\title{
Usage of a Bundle Application Process in Decreasing Ventriculoperitoneal Shunt Infections
}

\author{
Nimet ATES ${ }^{1}$, Ali KAFADAR ${ }^{2}$, Gokhan AYGUN³ ${ }^{3}$, Aytolan YILDIRIM ${ }^{4}$ \\ ${ }^{1}$ Istanbul University-Cerrahpasa, Cerrahpasa Faculty of Medicine, Department of Infection Control Comittee, Istanbul, Turkey \\ ${ }^{2}$ Istanbul University-Cerrahpasa, Cerrahpasa Faculty of Medicine, Department of Neurosurgery, Istanbul, Turkey \\ ${ }^{3}$ Istanbul University-Cerrahpasa, Cerrahpasa Faculty of Medicine, Department of Clinical Microbiology, Istanbul, Turkey \\ ${ }^{4}$ Istanbul University-Cerrahpasa, Florence Nightingale Faculty of Nursing, Department of Nursing Administration, Istanbul, Turkey \\ This study has been presented at the $16^{\text {th }}$ WFNS World Congress of Neurosurgery between 20 and 25 August 2017 at Istanbul, Turkey.
}

Corresponding author: Nimet ATES nimetates2011@gmail.com

\section{ABSTRACT}

AIM: To determine the effect of using three concurrent measures $(2 \%$ chlorhexidine gluconate $+70 \%$ alcohol for skin cleaning, changing the dressing every 48 hours, and observing the operating room) for patients who had venriculoperitoneal shunt (VPS) implantations on the infection rate.

MATERIAL and METHODS: This was a prospective interventional study. Using infection prevention compliance control lists, patients who had a VPS implanted between November 2012 and November 2013 were compared to those who had a VPS implanted between October 2011 and October 2012. The data included information about the patients, follow-up, and infection prevention. These data were obtained for the pre-, peri-, and postoperative periods.

RESULTS: Triple bundle prevention in the applied protocol significantly reduced the VPS infection rate in the study group compared to the control group $(\mathrm{p}<0.005)$.

CONCLUSION: These findings revealed the importance of creating a prevention protocol and following it regularly in a way for all surgery, operating room, and service teams fully comply to prevent VPS infections.

KEYWORDS: Ventriculoperitoneal shunt, Infection, Nursing, Bundle application, Skin antisepsis

\section{INTRODUCTION}

$\mathrm{V}$ entriculoperitoneal shunt surgery is a frequently used treatment for patients with hydrocephalus. Each year in the US, 70,000 patients are admitted to hospitals with complaints of hydrocephalus, approximately 40,000 undergo shunt surgery, and billions of dollars are spent on these patients (21). There are no epidemiological data related to hydrocephalus in Turkey.

Infection is a ventriculoperitoneal shunt complication that is a serious cause of shunt failure and is the fundamental reason for mortality and morbidity. The organisms that cause most shunt infections penetrate the patient's skin through wounds via surgical gloves or contaminated tools, reaching the patient's flora (9).

Skin antisepsis is considered to be a main component in wound infections. lodophors, chlorhexidine, and derivatives including these two agents are used most in surgery. Chlorhexidine gluconate is an important component that is usually used in an attempt to decrease infection risks associated with healthcare services. It is considered a safe, effective agent with wide clinical applications and has been used as a topical antiseptic for more than 50 years. Chlorhexidine gluconate has a rich activity spectrum, including Gram-positive and Gram-negative bacteria, ferments, and enveloped
Nimet ATEŞ (1D : 0000-0003-0417-3059 Ali KAFADAR (1) : 0000-0002-3312-3192
Gokhan AYGUN : (D): 0000-0001-6915-9843

Aytolan YILDIRIM (1) : 0000-0002-0475-6695 
viruses (10). Compared chlorhexidine+alcohol and povidone iodine on clean-contaminated wounds. A statistically significant decrease was observed for both superficial wounds and gashes in the chlorhexidine-alcohol group among theinfection rates (8). In a meta-analysis study (15), a comparison of chlorhexidine $(0.5 \%-4 \%)$ and povidone iodine $(0.7 \%-10 \%)$ was performed to improve incisional wound infection rates. In addition, in another meta-analysis study comparing chlorhexidine and povidone iodine for skin cleaning during clean and contaminated surgeries, it was found that chlorhexidine is effective in decreasing surgical and abdominal sepsis infections (18).

\section{MATERIAL and METHODS}

This was a prospective interventional study, aimed at determining the effect of bundled practices (using $2 \%$ chlorhexidine gluconate $+70 \%$ alcohol for skin cleaning, changing the dressing every 48 hours, and observing the operating room) for patients who had ventriculoperitoneal shunt implantations on the infection rate.

\section{Setting and Sample}

The study question was, "Do triple bundled applications prevent ventriculoperitoneal shunt infections?"

The study group comprised adult and pediatric patients who received a shunt for the first time in the Neurosurgery Clinic of a university hospital between November 2012 and November $2013(n=38)$. The control group comprised adult and pediatric patients who had a shunt for the first time a year before the study in the same hospital and clinic between November 2011 and November $2012(n=44)$.

\section{Sample Exclusion Criteria}

The following cases were excluded from the study:

(1) Cases with active meningitis and ventriculitis before the shunt surgery (verified by microbiological culture).

(2) Cases who had shunt placement at another hospital and came for revision.

(3) Cases who had shunt placement before November 2012 and applied for shunt revision.

(4) Cases who had shunt placement before November 2011 and applied for shunt revision.

\section{Data Collection Procedure}

Data were collected using the Patient Description and Observation Form (PDOF), which includes information and observations, and the Integrated Precautions Observation Form (IPOF), which includes information regarding infection prevention. The PDOF included 25 items regarding demographics, medical information, and patient observation, and the IPOF included 26 items under three main headings for patients in the control group. There were questions regarding observations about pre-/postsurgery preparation, team members, and patient dressing.

\section{Research Application Steps}

Operating room, intensive care, and service staff were interviewed and informed about the study steps, the precautions to be taken, and the things to do before the study.

The following are the application steps regarding patients in the study group. Patients in the study group received surgicalsite skin cleaning with $2 \%$ chlorhexidine gluconate $+70 \%$ alcohol and the dressings were not changed for 48 hours. The whole operation process was observed by a supervisor. The symptoms of infection, in addition to the laboratory and microbiology results, were followed up after the operations. Hand hygiene and symptoms of infection were explained to each patient / relative before discharge. Infection control surveillance for each patient continued over the phone by a physician for a year after the discharge.

The following are the application steps regarding patients in the control group. Patients in the control group were picked from those who had shunt placement through a list made from the data-processing system of the hospital. The data of these patients were obtained from discharge reports and other formal documents. Infection control surveillance was made over the phone after discharge.

Shunt infections were diagnosed according to the diagnosis criteria of the Centers for Disease Control and Prevention.

\section{Limitations of the Study}

- In total, three doses of cefazolin (1 g) were used as prophylaxis because of the different applications.

- The control group was selected retrospectively.

- Shunt placement surgeries was not considered to be an emergency; thus, the operating room was not available all the time.

- Asepsis requirements were not fulfilled because the anesthetist did not ensure hand hygiene and use proper gloves.

\section{Ethical Dimension of the Study}

Approval was obtained from the university's ethical committee (6.11.2012, A-31). The patients were informed of the aims and methods of the study. Adult patients signed consent forms, and parent consent was obtained for pediatric cases.

\section{Data Analysis}

The Kolmogorov-Smirnov distribution test was used to analyze normal distributions along with descriptive statistical methods (frequency, percentage, mean, and standard deviation). Pearson's chi-squared test and Fisher's exact test were used for study-control comparisons. The Mann-Whitney $U$ test was used to assess intergroup relation ships.

\section{RESULTS}

Table I shows the gender, age category, age group diagnosis, hydrocephalustype, presence or absence of an external ventricular drain (EVD), accompanying diseases, intensive 
Table I: Demographical Findings and Comparison Results of Study-Control Group Patients (Study Group N:38 - Control Group N: 44)

\begin{tabular}{|c|c|c|c|c|c|c|}
\hline & & \multicolumn{2}{|c|}{ Study } & \multicolumn{2}{|c|}{ Control } & \multirow{2}{*}{$\mathbf{p}$} \\
\hline & & $\mathbf{n}$ & $\%$ & $\mathbf{n}$ & $\%$ & \\
\hline \multirow{2}{*}{ Sex } & Female & 13 & 34.2 & 20 & 45.5 & \multirow{2}{*}{$\begin{array}{l}X^{2}=1.072 \\
p=0.209\end{array}$} \\
\hline & Male & 25 & 65.8 & 24 & 54.5 & \\
\hline \multirow{2}{*}{ Age Category } & Children & 17 & 44.7 & 20 & 45.5 & \multirow{2}{*}{$\begin{aligned} X^{2} & =0.004 \\
p & =0.563\end{aligned}$} \\
\hline & Adult & 21 & 55.3 & 24 & 54.5 & \\
\hline \multirow{4}{*}{ Age Group } & $0-6$ Months & 9 & 23.7 & 7 & 15.9 & \multirow{4}{*}{$\begin{array}{l}X^{2}=1.644 \\
p=0.649\end{array}$} \\
\hline & 6 Months - 2 Years & 4 & 10.5 & 6 & 13.6 & \\
\hline & 2-18 Years & 4 & 10.5 & 8 & 18.2 & \\
\hline & 18 Years and older & 21 & 55.3 & 23 & 52.3 & \\
\hline \multirow{5}{*}{ Diagnosis } & Primer Hydrocephalia & 17 & 44.7 & 12 & 27.3 & \multirow{5}{*}{$\begin{array}{l}X^{2}=3.850 \\
p=0.427\end{array}$} \\
\hline & Tumor & 10 & 26.3 & 11 & 25 & \\
\hline & Spina Bifida/ Meningomyelocele & 1 & 2.6 & 3 & 6.8 & \\
\hline & $\mathrm{SAH}$, Aneurism & 5 & 13.2 & 8 & 18.2 & \\
\hline & Normal pressure hydrocephalus NPH & 5 & 13.2 & 10 & 22.7 & \\
\hline \multirow{3}{*}{$\begin{array}{l}\text { Hydrocephalia } \\
\text { type }\end{array}$} & Obstructive & 11 & 28.9 & 15 & 34.1 & \multirow{3}{*}{$\begin{array}{l}X^{2}=4.895 \\
p=0.087\end{array}$} \\
\hline & Communicative & 23 & 60.5 & 29 & 65.9 & \\
\hline & Multiloculated & 4 & 10.5 & 0 & 0 & \\
\hline \multirow{2}{*}{ EVD/Ommaya } & Yes & 14 & 36.8 & 9 & 20.5 & \multirow{2}{*}{$\begin{array}{l}X^{2}=2.713 \\
p=0.100\end{array}$} \\
\hline & No & 24 & 63.2 & 35 & 79.5 & \\
\hline \multirow{2}{*}{$\begin{array}{l}\text { Accompanying } \\
\text { Diseases }\end{array}$} & Yes & 19 & 50 & 23 & 52.3 & \multirow{2}{*}{$\begin{array}{l}X^{2}=0.042 \\
p=0.837\end{array}$} \\
\hline & No & 19 & 50 & 21 & 47.7 & \\
\hline \multirow{2}{*}{ ICU stay } & Yes & 10 & 26.3 & 9 & 20.5 & \multirow{2}{*}{$\begin{array}{l}x^{2}=0.393 \\
p=0.530\end{array}$} \\
\hline & No & 28 & 73.7 & 35 & 79.5 & \\
\hline \multirow{2}{*}{ Survival } & Survives & 35 & 92.1 & 37 & 84.1 & \multirow{2}{*}{$\begin{array}{l}X^{2}=1.223 \\
p=0.223\end{array}$} \\
\hline & Death & 3 & 7.9 & 7 & 15.9 & \\
\hline
\end{tabular}

care unit stay, data, and comparison regarding the survival of patients in the study and control groups.

No significant difference was found between the groups in terms of all independent variables $(p>0.05)$. The minimum, maximum, and mean number of people in the surgical room during surgery for the study group were 4, 11, and 6 , respectively. Seven people were in the surgical room of the patient who had an infection. The minimum, maximum, and mean length of surgery for the study group were 30, 150, and 63 minutes, respectively. The length of surgery for the patient who had an infection was $30 \mathrm{~min}$ (Table II).

All patients who developed infections were male and children. Of the three children who had infections, two were in the sixmonth to one-year age group and the remaining one was in the one- to two-year age group. All three had communica- tive hydrocephalus, and their infections developed within zero to six months. Two of the reproducing microorganisms were methicillin-resistant coagulase-negative staphylococci: Candida albicans and methicillin-resistant Staphylococcus aureus. The three patients who developed infections had a fever. Two patients in the control group had fluid in the shunt end. The treatment method included removing the shunt, implanting an EVD, treatment with an antibiotic, and implanting a new shunt.

Of all the patients, $2.6 \%(1 / 38)$ of the study group and $6.8 \%$ $(3 / 44)$ of the control group had infections. One patient in the control group had an infection twice. On 1,000 shunt days, the rate of shunt infection was $0.61 \%$ in the study group and $7.63 \%$ in the control group. A significant difference was found between the study and the control groups in terms of shunt infection rates $(p=0.025, p<0.05)$. 
Table II: Preoperative and Intraoperative Patient Preparation and Work Team in the Study Group and Distribution of Wound Dressing Observations $(n=56)$

\begin{tabular}{|c|c|c|}
\hline & $\mathbf{n}$ & $\%$ \\
\hline \multicolumn{3}{|l|}{ Preoperative and Intraoperative Patient Preparation Observations } \\
\hline Preoperative skin cleaning was done using $2 \%$ chlorhexidine. & 56 & 100 \\
\hline Disposable razor was used for depilation. & 56 & 100 \\
\hline Someone appropriately traineddid the depilation. & 56 & 100 \\
\hline Wet depilation was made. & 56 & 100 \\
\hline Patient's skin was covered with a drape. & 56 & 100 \\
\hline Surgeries were performed as the first surgery in the morning. & 22 & 39.3 \\
\hline Shunt with antibiotic/antibiotic absorbed shunt was used. & 50 & 89.3 \\
\hline Vancomycin transition provided in antibiotic-impregnated shunt & 50 & 100 \\
\hline \multicolumn{3}{|l|}{ Preoperative and Intraoperative Work Team Observations } \\
\hline The whole team used masks covering their mouth and nose. & 56 & 100 \\
\hline The whole team wore bonnets covering their hair. & 56 & 100 \\
\hline The whole team took off their accessories. & 56 & 100 \\
\hline Hand washing took 3-5 minutes. & 56 & 100 \\
\hline Hand drying was performed correctly. & 56 & 100 \\
\hline Tools were opened in a sterile way. & 56 & 100 \\
\hline Shunt material was open at the end. & 50 & 89.3 \\
\hline Operating room door was kept closed. & 56 & 100 \\
\hline Anesthetist, doctor, nurse, and technician washed their hands before the operation. & 32 & 57.1 \\
\hline Anesthetist, doctor, nurse, and technician used gloves during the operation. & 22 & 39.3 \\
\hline Anesthetic was prepared aseptically. & 56 & 100 \\
\hline Arrivals and departures in theoperating room were kept to a minimum. & 56 & 100 \\
\hline The number of people in the operating room was kept to 5-7 people. & 50 & 89.3 \\
\hline Anesthesia table was kept away from the sterilized area. & 56 & 100 \\
\hline Movement around the surgery table was minimized. & 56 & 100 \\
\hline Prophylaxis was used during surgery. & 56 & 100 \\
\hline Team members were warned when necessary. & 56 & 100 \\
\hline \multicolumn{3}{|l|}{ Observations Regarding PostoperativeDressing } \\
\hline First dressing was placed 48 hours after the surgery. & 55 & 98.2 \\
\hline Dressing became dirty 6 . hour after the operation. & 1 & 1.8 \\
\hline Hands were washed before changing the dressing. & 56 & 100 \\
\hline Sterilized gloves were worn when applying the dressing. & 56 & 100 \\
\hline
\end{tabular}




\section{DISCUSSION}

The most effective factor in preventing shunt infections is hygienic skin preparation and a careful surgical technique. In this study, $2 \%$ chlorhexidine gluconate $+70 \%$ alcohol was used for skin antisepsis. We compared chlorhexidine + alcohol and povidone iodine treatment for contaminated wounds in a randomized experiment with 849 patients in six hospitals. A statistically significant decrease in the infection rate was observed for both superficial wounds and gashes in the chlorhexidine-alcohol group (8). In a meta-analysis comparing chlorhexidine and povidone iodine for skin cleaning during clean and contaminated surgeries, chlorhexidine was found to be effective in decreasing surgical and abdominal sepsis infections (18). In this study, we found that chlorhexidine was effective in decreasing infections. It was also found in a study that chlorhexidine and povidone iodine are the most effective agents for skin disinfection. Chlorhexidine showed the best residual activity among these agents (1).

In studies assessing the use of chlorhexidine for perioperative skin antisepsis, $3 \%$ parachlorometaxylenol, $0.7 \%$ povidone iodine $+74 \%$ isopropyl alcohol, and $2 \%$ chlorhexidine + $70 \%$ isopropyl alcohol were used during foot surgery. The chlorhexidine + alcohol combination significantly decreased the foot flora compared to other combinations (19). In another study on scapular surgery, $0.7 \%$ iodine $+74 \%$ isopropyl alcohol, $2 \%$ chlorhexidine $+70 \%$ alcohol, and $0.75 \%$ iodine scrub $+1 \%$ iodine application were compared. It was found in that study that the chlorhexidine + alcohol mixture decreased the staphylococcal skin bacteria at the incision area (23). In a randomized clinical study, $4 \%$ chlorhexidine and $10 \%$ povidone iodine were compared and it was found that chlorhexidine is more effective in decreasing the skin flora for 30 minutes (7). The study results were similar considering that $60 \%$ of the infectious agents were skin floral bacteria in shunt infections, although $4 \%$ chlorhexidine was not used and a decrease in floral bacteria was not considered. Moreover, it is recommended to use intraoperative skin preparations with an alcohol-based antiseptic agent unless contraindicated (4). Thus, $2 \%$ chlorhexidine $+70 \%$ alcohol was used to prevent surgical-site infections (SSIs) in our study.

Considering the result assessing studies for single or multiple precautions, the effect of single-application techniques on the incidence of shunt infections was assessed in 12 studies that were not multicentered. Many studies showed that surgical preparation is important (24). In a single-measure application, two studies showed significant differences between groups when the effect of depilation on decreasing the infection rate was assessed. It was found that shaving the scalp was not a critical step for the prevention of cerebrospinal fluid shunt infection $(12,20)$. It was found in a single application that using double gloves (the glove's exterior was taken off before implantation) decreased the infection rates compared to the normal control groups (25). In another single-application casecontrol study of 111 patients, double gloves were worn and the exterior of the glove was removed prior to handling the shunt. The infection rate in the control group was $16.33 \%$, whereas it was $3.77 \%$ in the double-gloved group. This was shown to decrease the infection rate (22). The no-touch principle was utilized, and the shunt tools and skin tools were separated to decrease contamination. In a double prevention study, these procedures decreased the infection rates three fold (11). In parallel with these studies, shunt infections decreased following the three applications in our study. A decrease in infections can be warranted through full compliance with single and multiple measures to prevent infections.

Preventative measures were used to eliminate infections and the effect of full compliance (zero tolerance) was assessed in infections in 126 patients who underwent 176 operations between January 1994 and January 2001 to eradicate postoperative shunt infections. Medical errors were not tolerated in any cases. This application was named "zero tolerance." The infection rate was $0.057 \%$ using this zerotolerance method. Zero-tolerance management in this study could not be administered for correct hand wash by the anesthesia team, correct glove use, and conducting surgeries as the first surgery in the morning (5).

Full compliance is very important in bundle applications to prevent SSIs. Compliance to a bundle program was assessed to prevent SSIs after colorectal surgery. The compliance rate increased from $10 \%$ to $60 \%$ and the SSI rate decreased by $36 \%$ (6). Full compliance with bundle applications had three parameters in our study. Full compliance was not guaranteed in other bundle applications. A study on prospective quality improving studies included appropriate antimicrobial prophylaxis, preoperative body temperature monitoring, blood sugar regulation, preoperative bowel preparation with antibiotics, performing a minimally invasive operation within the shortest time possible, and completing the operation in 100 minutes or less. The SSI development risk was $2 \%$ for full compliance to the six bundle items, whereas it was $17.5 \%$ for zero tolerance to one item. The study showed that the infection development risk in zero tolerance to bundle applications was less and that the risk of infection decreased when the number of bundled applications increased (26).

Another important subject is full compliance of healthcare professionals to bundled applications. Noncompliance of healthcare personnel to infection prevention processes is a complex subject. Effective strategies are needed to transform evidence-based studies and guidelines into effective applications conducted to prevent infections (27). Considering studies on healthcare personnel's compliance to bundled applications, full compliance in colorectal cancer surgery was successfully achieved by including key people from all healthcare personnel in the system, reviewing the study in monthly meetings under the chairmanship of a surgeon, and supporting the team (14). In other studies, it was found that including personnel from all healthcare staff and motivating them can provide full compliance to bundle applications $(2,13)$. In our study, the anesthesiology team's compliance regarding correct hand washing and glove use was low, which can be attributed to the fact that they did not actively participate in the team.

Considering the methods developed for effective applications to determine the factors affecting the compliance of 
healthcare personnel and to prevent infections, as well as the studies using this method, the Systems Engineering Initiative for Patient Safety model represents an approach oriented at innovative human factor engineering regarding patient safety. This model has a multi-interactional study system at its center, and it includes the person, duties, tools and technologies, physical environment, and organizational conditions. In a qualitative study under the guidance of this system, a work analysis was prepared regarding the prevention of Clostridium difficile, hand hygiene, and contact isolation. Obstacles including few, poorly located, and untidy sinks; lack of hot water; and not knowing where to wash hands negatively affected hand hygiene. Organizational factors significantly affect compliance to bundled approaches (27). This system is used to prevent C.difficile infections, assess obstacles and facilitators, and promote studies, and it is also helpful in assessing infection prevention practices and determining promoting opportunities (17). In a qualitative study using this system in India, where hospital infections are high, the facilitators and obstacles regarding hospital infection control applications were assessed (3). This system was also applied in another study and the obstacles were assessed. The results of this study showed that hospital infections could be prevented through a comprehensive work analysis, thereby maximizing the resources to overcome the known obstacles for infection control through a system engineering approach (16). This system can be used to assess the reasons why the anesthesiology team had low compliance to hand hygiene and surgeries were not the first surgeries in the morning.

\section{CONCLUSION}

In this study, triple bundle prevention in the applied protocol significantly reduced the shunt infection rate in the study group compared to the control group $(p<0.005)$. These findings and results revealed the importance of creating a prevention protocol and following it to prevent shunt infections.

Planned bundle precautions should be used to ensure the prevention of hospital infections. The low usage of bundle measures by health professionals is a matter to be resolved. A higher rate of using bundle precautions will yield lower infection rates.

\section{REFERENCES}

1. Ayliffe G, Babb J, Davies J, Lilly H: Hand disinfection: A comparison of various agents in laboratory and ward studies. Journal Hospital Infection 11: 226-243, 1988

2. Ban KA, Minei JP, Laronga $C$, Harbrecht BG, Jensen $E H$, Fry DE, Itani KM, Dellinger EP, Ko CY, Duane TM: American college of surgeons and surgical infection society: Surgical site infection guidelines, 2016 update. J Am Coll Surg 224: 59-77, 2017

3. Barker A, Brown K, Siraj D, Ahsan M, Sengupta S, Safdar N: Barriers and facilitators to infection control at a hospital in northern india: a qualitative study. Antimicrobial Resistance and Infection Control 6:2-7, 2017
4. Berríos-Torres SI, Umscheid CA, Bratzler DW, Leas B, Stone EC, Kelz RR2, Reinke CE, Morgan S, Solomkin JS, Mazuski JE, Dellinger EP, Itani KMF, Berbari EF, Segreti J, Parvizi J, Blanchard J, Allen G, Kluytmans JAJW, Donlan R, Schecter WP; Healthcare Infection Control Practices Advisory Committee: Centers for disease control and prevention guidline for the prevention of surgical site infection. JAMA Surg 152:784-791, 2017

5. Choksey MS, Malik IA: Zero tolerance to shunt infections: Can it be achieved? J Neurol Neurosurg Psychiatry 75:87-91, 2004

6. Crolla RM, van der Laan L, Veen EJ, Hendriks Y, van Schendel C, Kluytmans J: Reduction of surgical site infections after implementation of a bundle of care. PLoS One 7:1-6, 2012

7. Culligan PJ, Kubik K, Murphy M, Blackwell L, Snyder J: A Randomized trial that compared povidone iodine and chlorhexidine as antiseptics for vaginal hysterectomy. American Journal of Obstetrics and Gynecolog 192:422-425, 2005

8. Darouiche RO, Wall MJ Jr, Itani KM, Otterson MF, Webb AL, Carrick MM, Miller HJ, Awad SS, Crosby CT, Mosier $\mathrm{MC}$, Alsharif A, Berger DH: Chlorhexidine-Alcohol versus povidone-iodine for surgical-site antisepsis. The New England Journal of Medicine 362:18-26, 2010

9. Drake JM, Saint Rose C: Şant Kitabı. İstanbul: Nobel Tıp Kitabevleri, 2002

10. Edmiston CE, Bruden B, Rucinski MC, Henen C, Graham MB, Lewis BL: Reducing the risk of surgical site infections: Does chlorhexidine gluconate provide a risk reduction benefit? American Journal of Infection Control 41:49-55, 2013

11. Faillace WJ: A No-touch technique protocol to diminish cerebrospinal fluid shunt infection. Surg Neurol 43:344-350, 1995

12. Horgan MA, Piatt JH Jr: Shaving of the scalp may increase the rate of infection in csf shunt surgery. Pediatr Neurosurg 26: 180-184, 1997

13. Itani KM: Care bundles and prevention of surgical site infection in colorectal surgery. JAMA 314: 289-290, 2015

14. Keenan JE, Speicher PJ, Thacker JKM, Walter M, Kuchibhatla M, Mantyh CR: The preventive surgical site infection bundle in colorectal surgery an effective approach to surgical site infection reduction and health care cost savings. JAMA Surg 149:1045-1052, 2014

15. Lee I, Agarwal RK, Lee BY, Fishman NO, Umscheid CA: Systematic review and cost analysis comparing use of chlorhexidine with use of iodine for preoperative skin antisepsis to prevent surgical site infection. Infection Control \& Hospital Epidemiology 31:1219-1229, 2010

16. Mitchell K, Barker A, Abad CL, Safdar N: Infection control at an urban hospital in manila, philippines: A systems engineering assessment of barriers and facilitators. Antimicrobial Resistance and Infection Control 6: 2-9, 2017

17. Ngam C, Hundt AS, Haun N, Carayon P, Stevens L, Safdar $\mathrm{N}$ : Barriers and facilitators to clostridium difficile infection prevention. A nursing perspective. American Journal of Infection Control 45:1363-1368, 2017 
Ates N. et al: Ventriculoperitoneal Shunt Infections

18. Noorani A, Rabey N, Walsh SR, Davies RJ: Systematic review and meta-analysis of preoperative antisepsis with chlorhexidine versus povidone-iodine in clean-contaminated surgery. British Journal Surgery 97:1614-1620, 2010

19. Ostrander RV, Botte MJ, Brage ME: Efficacy of surgical preparation solutions in foot and ankle surgery. Journal of Bone Joint Surgery 87:980-985, 2005

20. Ratanalert S, Musikawat P, Oearsakul T, Saeheng S, Chowchuvech V: Nons haved ventriculoperitoneal shunt in Thailand. Journal of Clinical Neuroscience 12:147-149, 2005

21. Reddy GK, Bollam P, Caldito G: Ventriculoperitoneal shunt surgery and the risk of shunt infection in patient with hydrocephalus: Long term single institution experience. World Neurosurgery 10:1-9, 2012

22. Rehman AU, Rehman TU, Bashir HH, Gupta VA: Simple method to reduce infection of ventriculoperitoneal shunts. J Neurosurg Pediatrics 5:569-572, 2010

23. Saltzman MD, Nuber GW, Gryzlo SM, Marecek GS, Koh JL: Efficacy of surgical preparations in shoulder surgery. Journal Bone Joint Surgery Am 91:1949-1953, 2009
24. Sarmey N, Kshettry VR, Shriver MF, Habboub G, Machado AG, Weil RJ: Evidence-based interventions to reduce shunt infections: A systematic review. Childs Nerv Syst 31:541-549, 2015

25. Tulipan N, Cleves MA: Effect of an intraoperative double gloving strategy on the incidence of cerebrospinal fluid shunt infection. J Neurosurg 104:5-8, 2006

26. Waits SA, Fritze D, Banerjee M, Zhang W, Kubus J, Englesbe MJ, Campbell DA Jr, Hendren S: Developing an argument for bundled interventions to reduce surgical site infection in colorectal surgery. Surgery 155:602-606, 2014

27. Yanke E, Moriarty H, Carayon P, Safdar N: A qualitative, interprofessional analysis of barriers to and facilitators of implementation of the Department of Veterans Affairs' Clostridium difficile prevention bundle using a human factors engineering approach. Am J Infect Control 46:276-284, 2018 